\title{
Mudanças na estrutura do solo avaliada com uso de tomografia computadorizada
}

\author{
Luiz Fernando Pires ${ }^{(1)}$ e Osny Oliveira Santos Bacchi(2)
}

\begin{abstract}
(1)Universidade Estadual de Ponta Grossa, Departamento de Física, Avenida Carlos Cavalcanti, № 4.748, Uvaranas, CEP 84030-900 Ponta Grossa, PR. E-mail: Ifpires@uepg.br (2)Universidade de São Paulo, Centro de Energia Nuclear na Agricultura, Caixa Postal 96, CEP 13400-970 Piracicaba, SP. E-mail: osny@cena.usp.br
\end{abstract}

\begin{abstract}
Resumo - O objetivo deste trabalho foi avaliar, em escala milimétrica, a modificação da densidade e da porosidade de amostras deformadas de solo submetidas a ciclos de umedecimento e secamento (U-S), por meio da tomografia computadorizada de raios gama. Amostras com $98,1 \mathrm{~cm}^{3}$ foram preparadas procedendo ao peneiramento do solo em malha de $2 \mathrm{~mm}$ e acondicionamento de forma homogênea em tubos de PVC. As amostras de solo foram submetidas a um, dois e três ciclos de U-S. As amostras controle não foram submetidas a nenhum ciclo de U-S. Após a aplicação dos ciclos de U-S, a porosidade das amostras diminuiu e as camadas de solo se adensaram. Os dados de tomografia computadorizada permitiram análise contínua da densidade do solo e de sua porosidade, em camadas milimétricas $(0,08 \mathrm{~cm})$, o que não pode ser alcançado facilmente por métodos tradicionais usados em física do solo.
\end{abstract}

Termos para indexação: análise de imagens, atenuação de raios gama, ciclos de umedecimento e secamento, densidade do solo, porosidade do solo.

\section{Soil structure changes evaluated with computed tomography}

\begin{abstract}
The objective of this work was to evaluate in millimetric scale changes in soil bulk density and porosity, using the gamma-ray computed tomography in soil samples with disturbed structure due to wetting and drying (W-D) cycles. Soil samples with $98.1 \mathrm{~cm}^{3}$ were sieved using a $2 \mathrm{~mm}$ mesh and homogenously packed in PVC cylinders. Soil samples were submitted to 1, 2, and 3 W-D cycles. Control samples were not submitted to W-D cycles. After repetitions of W-D cycles, soil sample porosity decreased and soil layers became denser. Computed tomography allowed a continuous analysis of soil bulk density and also soil porosity along millimetric $(0.08 \mathrm{~cm})$ layers, what cannot be provided by traditional methods used in soil physics.
\end{abstract}

Index terms: image analysis, gamma-ray attenuation, wetting and drying cycles, soil bulk density, soil porosity.

\section{Introdução}

$\mathrm{Na}$ ciência do solo, os pioneiros no uso da tomografia computadorizada foram Petrovic et al. (1982), Hainsworth \& Aylmore (1983) e Crestana et al. (1985), que utilizaram essa técnica para medir a densidade do solo $\left(\rho_{\mathrm{s}}\right)$, a distribuição espacial do conteúdo de água e a retenção e movimento da água no solo. Os primeiros resultados de pesquisa publicados foram obtidos com tomógrafos computadorizados médicos. No entanto, o preço desses instrumentos tornava proibitiva a pesquisa aplicada com uso dessa técnica. Cesareo \& Giannini (1980) demonstraram ser possível construir tomógrafos computadorizados mais simples e baratos, denominados de primeira geração, que poderiam ser usados em pesquisas em outras áreas de conhecimento além da medicina.
No Brasil, trabalhos desenvolvidos na Embrapa Instrumentação Agropecuária, no Centro de Energia Nuclear na Agricultura, e na Universidade Federal de Pelotas são exemplos do uso desses equipamentos em ciência do solo (Macedo et al., 1998; Pedrotti et al., 2003; Pires et al., 2007).

Atualmente o uso de tomógrafos computadorizados de segunda e terceira gerações, com resolução micrométrica, tem sido comum. No entanto, um problema que envolve o uso desses equipamentos refere-se ao tamanho da amostra a ser analisada. Geralmente, em razão das características das fontes de raios $\mathrm{X}$, as amostras não devem exceder alguns poucos centímetros. Sendo assim, embora os tomógrafos computadorizados de raios gama forneçam apenas imagens com resolução milimétrica, a sua vantagem

Pesq. agropec. bras., Brasília, v.45, n.4, p.391-400, abr. 2010 
reside no fato de ser possível trabalhar com amostras com dimensões superiores a $10 \mathrm{~cm}$ de comprimento, utilizando-se fontes de ${ }^{137} \mathrm{Cs}$. Esses tomógrafos, no entanto, não possibilitam a investigação de distribuições de tipos e tamanhos de poros. Possibilitam apenas inferir sobre modificações da porosidade $(\varphi)$ da amostra, com base em valores médios de $\rho_{\mathrm{s}}$. A grande vantagem da técnica é ser não invasiva e permitir análises detalhadas de perfis de $\rho_{\mathrm{s}} \mathrm{e} \varphi$. Mudanças na estrutura do solo por processos naturais ou atividades agropecuárias podem ser investigadas com sucesso com uso de sistemas tomográficos. O fato de ser uma técnica não destrutiva possibilita avaliar modificações temporais da estrutura do solo em uma mesma amostra, o que não é possível com técnicas tradicionais de análise de imagens como a micromorfologia.

Ciclos de umedecimento e secamento (U-S) representam um exemplo de processo natural que pode causar modificações da estrutura do solo, uma vez que eles afetam a distribuição das partículas e, consequentemente, dos poros do solo. Modificações na distribuição do tamanho de poros por ciclos sucessivos de U-S afetam diretamente a distribuição espacial e temporal da umidade do solo, com importantes consequências práticas para estudos em irrigação (Tessier et al., 1990; Pardini et al., 1996; Hussein \& Adey, 1998; Li et al., 2004).

O objetivo deste trabalho foi avaliar em escala milimétrica a modificação da densidade $\left(\rho_{\mathrm{s}}\right)$ e da porosidade $(\varphi)$ de amostras deformadas de solo submetidas a repetidos ciclos de U-S, por meio da tomografia computadorizada de raios gama .

\section{Material e Métodos}

O solo usado para preparo das amostras foi coletado na camada superficial $(0-10 \mathrm{~cm})$, em área experimental da Escola Superior de Agricultura Luiz de Queiroz, e foi caracterizado como Nitossolo Vermelho Eutrófico (NVe) (Santos et al., 2006). Na camada amostrada, foram determinados $240 \mathrm{~g}$ de areia, $330 \mathrm{~g}$ de silte, $430 \mathrm{~g}$ de argila e 20,2 $\mathrm{g}$ de matéria orgânica por quilograma de solo.

O solo coletado foi secado ao ar e passado em peneira com malha de $2 \mathrm{~mm}$, e homogeneamente acondicionado a tubos de PVC com 5,0 cm de altura e $5,0 \mathrm{~cm}$ de diâmetro. No processo de preenchimento dos cilindros, a cada $0,5 \mathrm{~cm}$ de altura, pequenos impactos foram feitos manualmente nos cilindros, por um minuto, com o intuito de promover uma melhor acomodação dos agregados e partículas do solo nos espaços vazios. Foram preparadas três amostras, com volume aproximado de $98,1 \mathrm{~cm}^{3}$, para serem submetidas aos ciclos de U-S.

O processo de umedecimento escolhido foi por ascensão capilar, usado durante a obtenção de curvas de retenção da água no solo (Reichardt \& Timm, 2004). O procedimento de umedecimento consistiu, inicialmente, em se manter $0,3 \mathrm{~cm}$ de água a partir da base dos cilindros com solo, durante duas horas. Após este período, igual quantidade de água foi acrescentada a cada duas horas até que um nível de poucos milímetros abaixo do topo do cilindro fosse alcançado. Quarenta e oito horas foram necessárias para se obter $\mathrm{o}$ umedecimento das amostras sem o aprisionamento de bolhas de ar.

Para a secagem das amostras, elas foram colocadas sobre peneira com malha de $2 \mathrm{~mm}$ até que atingissem aproximadamente a massa correspondente à do solo secado ao ar (amostras controle). As amostras de solo foram submetidas a um (1C), dois (2C) e três (3C) ciclos de U-S. As amostras controle (0C) não foram submetidas a nenhum ciclo de U-S.

Durante a tomografia de uma amostra de solo, cada ponto da amostra de solo varrido pelo feixe de raios gama resulta na atenuação da radiação emitida de acordo com o coeficiente de atenuação linear $(\mu)$ do material amostrado. $\mathrm{O}$ grau de atenuação da radiação está estreitamente relacionado ao valor da unidade tomográfica (UT) gerado na tomografia da amostra (Macedo et al., 1998). No solo, como os valores de $\mu$ variam para cada caminho cruzado pelo feixe de radiação, a UT varia de acordo com as contribuições de partículas minerais, matéria orgânica, água e ar. Usualmente, a atenuação causada pelo ar contido no solo é insignificante quando comparada com a provocada pelas partículas e água do solo (Ferraz \& Mansell, 1979). Dessa forma, diferenças entre os valores de UT associados aos pontos que compõem a imagem de seção transversal do solo correspondem a variações da atenuação da radiação gama pelo solo e água.

Na imagem tomográfica, regiões escuras representam pontos com os mais altos valores de $\mu$, e regiões claras com os mais baixos. Variações entre essas duas regiões 
estão associadas a diferenças na densidade e no teor de água da amostra.

O tomógrafo utilizado tem sistema de varredura de primeira geração de raios gama, com arranjo fonte e detector fixos. A fonte de raios gama $\left({ }^{241} \mathrm{Am}\right)$ emite fótons com uma energia de $59,54 \mathrm{keV}$ e possui atividade de aproximadamente 3,7 GBq. A detecção dos fótons gama foi feita por um detector de cintilação sólida de $\mathrm{NaI}(\mathrm{Tl})$ do tipo plano com dimensões de 7,62x7,62 $\mathrm{cm}$. Na saída da fonte e na entrada do detector, foram fixados colimadores de chumbo de 1 e $3 \mathrm{~mm}$ de diâmetro, respectivamente, para evitar que fótons espalhados de menor energia (secundários) fossem detectados. Os passos angulares para as amostras de solo utilizadas neste experimento foram de $2,25^{\circ}$, até que se alcançasse uma rotação completa de $180^{\circ}$. Os passos lineares foram fixados em $0,08 \mathrm{~cm}$ para a matriz selecionada de $80 \times 80$ pontos, para se obter a melhor resolução possível. Detalhes da descrição e funcionamento de equipamentos similares ao usado neste estudo podem ser encontrados em Crestana et al. (1992) e Cruvinel \& Crestana (1996).

$\mathrm{Na}$ reconstrução das imagens tomográficas, foi utilizado o programa Microvis (2000), desenvolvido pela Embrapa Instrumentação Agropecuária. No caso das imagens tomográficas obtidas neste trabalho, a escala de cinza está invertida, uma vez que, no programa Microvis, ao preto é atribuido o menor valor de atenuação. A calibração do sistema tomográfico foi obtida da correlação linear entre os $\mu$ e as UTs da água, etanol, acrílico, nylon e glicerina (Crestana et al., 1992). As amostras de água, etanol e glicerina utilizadas para calibração foram acondicionadas em caixas vedadas de acrílico com borda de $0,4 \mathrm{~cm}$ e dimensões de $8,0 \times 8,0 \times 8,0 \mathrm{~cm}$. Os materiais sólidos acrílico e nylon eram peças cúbicas.

Nas medidas de $\mu$ das amostras usadas na calibração, selecionaram-se cinco regiões diferentes das amostras para serem cruzadas pelo feixe de radiação, e três repetições de contagens foram realizadas para cada região, durante 1 minuto. Para cada região, foi calculado o $\mu$, e seu valor final representa a média aritmética simples das cinco regiões varridas pelo feixe. O mesmo procedimento foi seguido para o cálculo do valor médio de $\mu$ para as amostras de solo estudadas.

As imagens tomográficas das amostras de solo foram obtidas em cortes transversais, com área de $19,63 \mathrm{~cm}^{2}$ e volume de $1,96 \mathrm{~cm}^{3}$, aproximadamente. $O$ feixe passou ao longo da região central das amostras, ou seja, à altura de $2,5 \mathrm{~cm}$ (Figura 1). No cálculo do volume, foi considerado um valor de $1 \mathrm{~mm}$ (colimador na fonte) para a espessura da fatia de solo abrangida pela tomografia. Para as amostras controle (0C) e os tratamentos $1 \mathrm{C}, 2 \mathrm{C}$ e $3 \mathrm{C}$, os cortes tomográficos foram obtidos sempre na mesma posição, com a finalidade de comparar os efeitos temporais dos ciclos de U-S na mesma região da amostra.

As tomografias foram realizadas para valores praticamente constantes de umidade do solo $(\theta)$ para evitar variações nas imagens por diferenças na umidade residual $\left(\theta_{\mathrm{r}}\right)$ após cada tratamento. Quando as amostras alcançaram este valor residual, elas foram envolvidas em filme plástico, para minimizar uma possível perda ou ganho de água durante as tomografias.

$\mathrm{Na}$ análise quantitativa das imagens em 2D, foram selecionadas áreas retangulares contendo 1.880 dados de UT, na matriz de dados referente a cada imagem das amostras de solo (Figura 1). A dimensão da matriz de dados ( $47 \mathrm{x} 40$ pontos) foi escolhida com o intuito de se obter uma área representativa da amostra (Baveye et al., 2002), distante das bordas, para evitar interferência, nas imagens tomográficas, das interfaces PVC-solo-ar (Herman, 1980). Cada pixel da matriz,

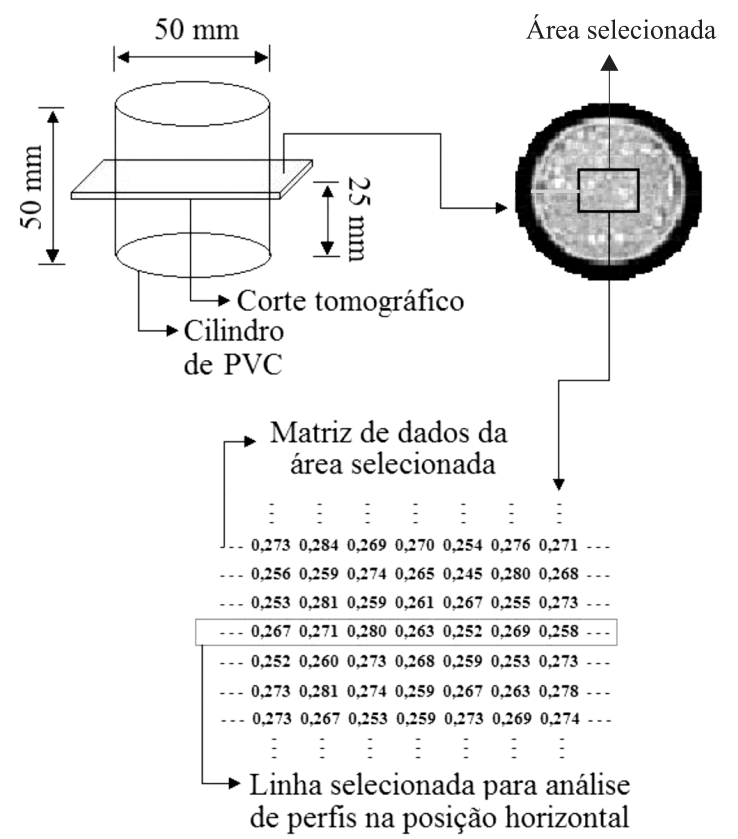

Figura 1. Diagrama esquemático dos cilindros que continham as amostras de solo e matriz de dados de unidades tomográficas (UT) e linha da matriz selecionada para o estudo dos perfis destas propriedades. 
referente a um valor absoluto de UT, possuía dimensões de $0,0064 \mathrm{~cm}^{2}$.

As médias aritméticas de UT para cada área (matriz $47 \times 40$ pontos) foram calculadas e convertidas em valores de $\rho_{\mathrm{s}}\left(\mathrm{g} \mathrm{cm}^{-3}\right)$, por meio da equação,

$$
\rho_{\mathrm{s}}=\left[(\mathrm{UT} / \alpha)-\mu_{\mathrm{mag}} \theta_{\mathrm{r}}\right] / \mu_{\mathrm{ms}},
$$

em que: $\mu_{\mathrm{ms}}$ e $\mu_{\mathrm{mag}}\left(\mathrm{cm}^{2} \mathrm{~g}^{-1}\right)$ são os coeficientes de atenuação de massa do solo e da água, respectivamente, e $\alpha$ é o coeficiente angular entre $\mu \mathrm{eUT}$, obtido a partir da calibração do tomógrafo.

Os perfis de $\rho_{\mathrm{s}}$ foram obtidos a partir da seleção de uma linha, contendo 50 dados de UT, da matriz de dados (Figura 1). A linha foi selecionada no centro da matriz de dados, para todas as imagens, e os valores de $\rho_{\mathrm{s}}$ para cada tratamento representam médias de três valores, em virtude das repetições.

Para a medida da porosidade do solo, foi utilizada a equação,

$$
\phi(\%)=100\left(1-\rho_{\mathrm{s}} / \rho_{\mathrm{p}}\right)
$$

em que: $\rho_{\mathrm{p}}\left(\mathrm{g} \mathrm{cm}^{-3}\right)$ representa a densidade de partículas do solo.

Com a finalidade de se comparar o efeito dos ciclos de U-S na estrutura das amostras de solo, foram realizados cálculos do desvio relativo (DR) entre o tratamento $0 \mathrm{C}$ e os demais. O DR foi calculado como,

$$
\operatorname{DR}(\%)=100\left[\left(\mathrm{X}_{\mathrm{iC}}-\mathrm{X}_{0 \mathrm{C}}\right) / \mathrm{X}_{0 \mathrm{C}}\right] \text {, }
$$

em que: X é a porosidade ou densidade das amostras e o termo i varia de 1 até 3.

\section{Resultados e Discussão}

Os valores de $\mu_{\mathrm{ms}}$ foram $0,363 \pm 0,003 \mathrm{~cm}^{2} \mathrm{~g}^{-1}$ e os de $\mu_{\text {mag }} 0,199 \pm 0,003 \mathrm{~cm}^{2} \mathrm{~g}^{-1}$. A inclinação da reta de calibração do tomógrafo computadorizado (TC) entre UT e $\mu$ foi 0,955 , e o coeficiente de correlação de Pearson (r) foi 0,99 , com correlação positiva alta entre os dados experimentais. Esse valor de $r$ mostra uma boa concordância entre UT e $\mu$, e é um resultado relevante para a obtenção de imagens tomográficas de boa qualidade (Crestana et al., 1985).

Imagens de seções transversais referentes às amostras $0 \mathrm{C}$ até $3 \mathrm{C}$ permitiram uma análise qualitativa das mudanças na estrutura do solo após os ciclos de
U-S (Figura 2). Os pontos escuros representam os valores máximos de $\rho_{\mathrm{s}}$ e os pontos mais claros o oposto. Ressalte-se que, em razão da resolução milimétrica do $\mathrm{TC}$, cada valor de $\rho_{\mathrm{s}}$ e $\varphi$ representa pequenas porções de área de 0,08x0,08 $\mathrm{cm}$ da amostra de solo. Por esse motivo, optou-se por chamar $\varphi$ de porosidade pela imagem, pois a resolução do método, neste caso em particular, não permite discriminar poros de partículas. Os altos valores de $\rho_{\mathrm{s}}$ próximos às bordas das amostras, representadas numa imagem similar a um anel externo escuro, devem-se a atenuação dos fótons pelas paredes do cilindro de PVC. O valor máximo de $\rho_{\mathrm{s}}$ nas amostras analisadas foi de $1,65 \mathrm{~g} \mathrm{~cm}^{-3}$.

Entre as vantagens da tomografia computadorizada estão o fato de esse procedimento permitir análises não destrutivas das amostras de solo e a ausência da necessidade de qualquer espécie de processamento das amostras. Outras técnicas tradicionalmente usadas, tais como a análise micromorfológica e porosimetria de mercúrio, não
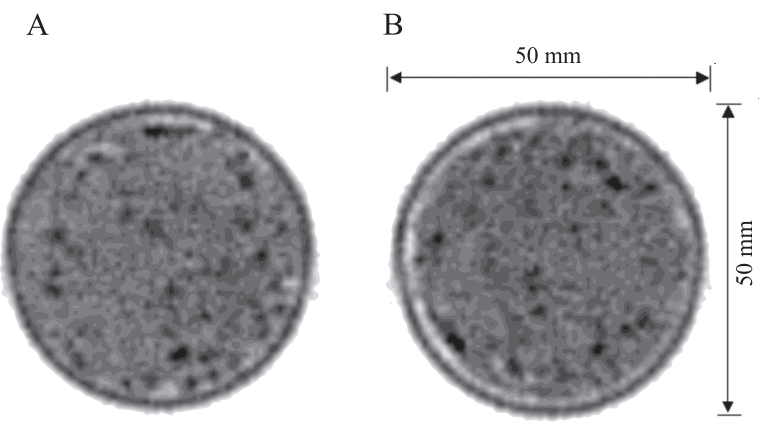

C

$\mathrm{D}$

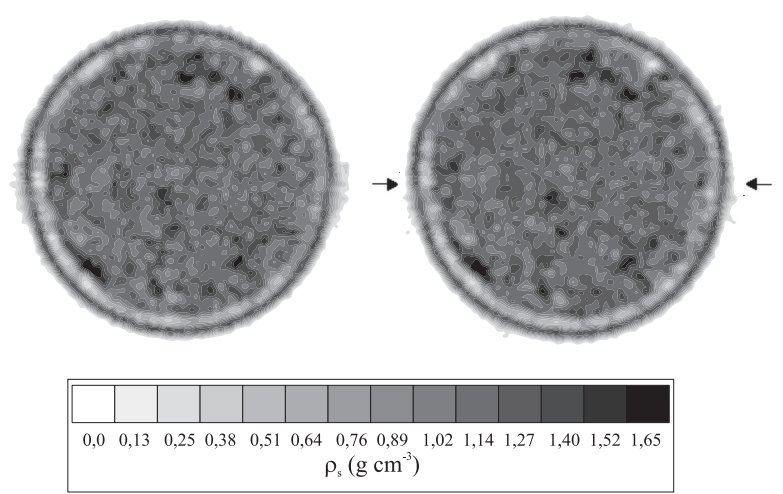

Figura 2. Densidades do solo $\left(\rho_{\mathrm{s}}\right)$ obtidas por meio de imagens tomográficas de uma seção transversal de amostras de solo produzidas artificialmente e submetidas a: A, nenhum ciclo de umedecimento e secamento (U-S); B, um ciclo de U-S; C, dois ciclos de U-S; C, D, três ciclos de U-S. A seta indica a posição selecionada na amostra para análise dos perfis de densidade do solo $\left(\rho_{\mathrm{s}}\right)$. 
possibilitam medidas do sistema poroso do solo em uma mesma amostra por serem metodologias destrutivas. A tomografia computadorizada também permite a obtenção de imagens em um tempo relativamente curto, mesmo em sistemas de primeira geração (horas), quando comparada com a micromorfologia (semanas ou meses). Sistemas de segunda geração são capazes de gerar imagens em 3D, com resoluções micrométricas e aquisição da imagem em minutos, sendo ferramentas interessantes para uma melhor caracterização do solo (Tippkotter et al., 2009).

Como as imagens tomográficas fornecem análises apenas qualitativas, foram realizados estudos quantitativos das amostras por meio de análises da matriz de dados de $\rho_{\mathrm{s}}$ (Figura 3) e de $\varphi$ (Figura 4).

Nos perfis de $\rho_{\mathrm{s}}$, é possível observar que os ciclos de U-S causam mudanças importantes na sua distribuição para a região da matriz de dados de UT escolhida (Figura 3). Pela análise dos perfis de $\rho_{\mathrm{s}}$, é possível concluir que essa propriedade física do solo apresenta uma tendência de aumento, quando as amostras $0 \mathrm{C}$ e as demais são comparadas (Figuras $3 \mathrm{~A}, \mathrm{C}$ e E).

Na comparação dos tratamentos $0 \mathrm{C}$ e $1 \mathrm{C}$ (Figura $3 \mathrm{~B}$ ), observa-se que um único ciclo de U-S já é suficiente para ocasionar o aumento de $\rho_{\mathrm{s}}$. Isto é verificado no gráfico pelo aumento de valores de DR positivos. Comportamento semelhante é observado na comparação entre $0 \mathrm{C}$ e $2 \mathrm{C}$ (Figura $3 \mathrm{D}$ ), o que indica que as modificações causadas por $1 \mathrm{C}$ e $2 \mathrm{C}$ em $0 \mathrm{C}$ são praticamente as mesmas. No entanto, o maior efeito sobre a densidade das amostras de solo ocorreu com a aplicação de três ciclos de U-S (Figura $3 \mathrm{~F}$ ). Isto pode facilmente ser verificado pela maior escassez de valores de DR negativos no gráfico que compara $0 \mathrm{C}$ e $3 \mathrm{C}$. Para este caso, praticamente todos os valores de $\rho_{\mathrm{s}}$ médio das linhas das matrizes analisadas apresentaram aumento com os ciclos de U-S.

Os valores de $\rho_{\mathrm{s}}$ médio encontrados para os diferentes tratamentos, usando-se para o cálculo a média de uma matriz com 1.880 dados de UT, foram 1,15, 1,21, 1,22 e $1,23 \mathrm{~g} \mathrm{~cm}^{-3}$ para os tratamentos $0 \mathrm{C}, 1 \mathrm{C}, 2 \mathrm{C}$ e $3 \mathrm{C}$, respectivamente. A tomografia computadorizada permitiu a obtenção de valores de $\rho_{\mathrm{s}}$ para fatias com $0,08 \mathrm{~cm}$ de espessura, o que possibilitou um estudo bastante detalhado da variação de $\rho_{\mathrm{s}}$ com os tratamentos.

Uma possível explicação para o aumento de $\rho_{\mathrm{s}}$ seria o assentamento das partículas de solo orientadas paralelamente após os ciclos de U-S, uma vez que as amostras usadas eram deformadas. Portanto, já durante o primeiro umedecimento, a aproximação das partículas e agregados do solo pode ter feito com que a massa de solo sofresse uma pequena contração com redução do volume da amostra. Isto explicaria o aumento da densidade do solo com os ciclos. Viana et al. (2004), trabalhando também com amostras deformadas, encontraram mudanças significativas na estrutura do solo após a aplicação de ciclos de U-S. Segundo estes autores, as alterações observadas podem ser atribuídas a um mecanismo de acomodação e rearranjo das partículas do solo após os ciclos de U-S.

Um outro processo que pode ocorrer é o adensamento do solo causado por deformações plásticas, em virtude da migração de argilas durante os ciclos de U-S (Vidal-Torrado et al., 1999). Os materiais mais finos das amostras, em virtude dos ciclos de U-S, tendem a ser carreados para as camadas mais profundas durante o secamento, preenchendo parte dos poros das camadas inferiores. Como as amostras eram deformadas, as partículas mais finas do solo estariam mais dispersas, o que favoreceria sua eluviação no processo de secamento. Segundo Silva \& Cabeda (2005), o processo de deposição de argila nas paredes dos poros e sobre grãos de quartzoocasiona um arranjo mais compacto de partículas, e aumenta o adensamento do solo.

A análise da distribuição de $\varphi$ mostra que, com a aplicação dos ciclos de U-S, ocorre uma tendência de aumento na frequência de menores valores (Figura 4). Essa tendência pode ser verificada nos gráficos que comparam o tratamento $0 \mathrm{C}$ com os demais (Figura $4 \mathrm{~A}, \mathrm{C}$ e E). $\mathrm{Na}$ análise de DR para as amostras $0 \mathrm{C}$ e $1 \mathrm{C}$ (Figura 4B), é possível observar um grande aumento (valores positivos de DR) da $\varphi$ pela aplicação do ciclo de U-S, nas faixas de $31-39 \%$ e $71-75 \%$. No intervalo de $55-71 \%$, os valores de $\varphi$ foram superiores para $0 \mathrm{C}$ em comparação a $1 \mathrm{C}$, com maiores $\mathrm{DR}$ no intervalo de porosidade $59-67 \%$. Na aplicação de dois ciclos de U-S (Figura 4 D), ocorre um aumento ainda maior de $\varphi$ nas faixas de $31-39 \%$ e $71-75 \%$ em comparação com as amostras 0C. Novamente, no intervalo de $55-71 \%$, os valores de $\varphi$ foram superiores para $0 \mathrm{C}$ em comparação a $1 \mathrm{C}$ com maior DR de $-57 \%$ para o intervalo de 59-63\%. Após a aplicação de três ciclos de U-S, verificaram-se as mesmas alterações de $\varphi$ já observadas para $1 \mathrm{C}$ e $2 \mathrm{C}$ em relação à $0 \mathrm{C}$ (Figura $4 \mathrm{~F}$ ). As maiores alterações de $\varphi$ comparando $0 \mathrm{C}$ e 3C também ocorreram nas faixas de $31-39 \%$ e $71-75 \%$. Novamente no intervalo de $55-71 \%$, os valores de $\varphi$ foram superiores para $0 \mathrm{C}$ em comparação a $3 \mathrm{C}$ com maior DR para o intervalo de $63-67 \%$. 
É possível observar que, mesmo o uso de apenas um ciclo de U-S já causa grandes mudanças na estrutura do solo, e a semelhança dos gráficos após aplicação dos demais ciclos ratificam esse resultado (Figura 4). Este
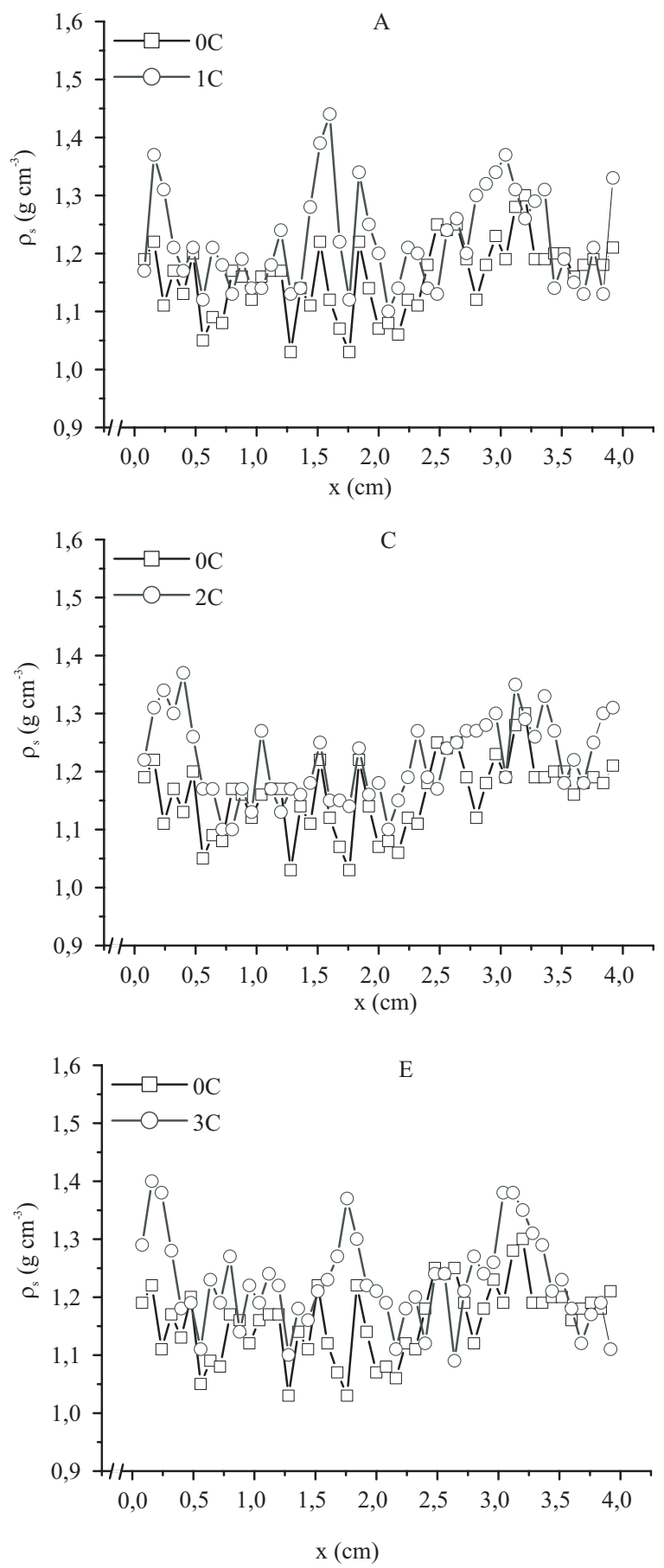

resultado demonstra que podem ocorrer mudanças importantes na estrutura de amostras de solo produzidas artificialmente em razão de ciclos de U-S. Estas modificações podem se tornar extremamente relevantes
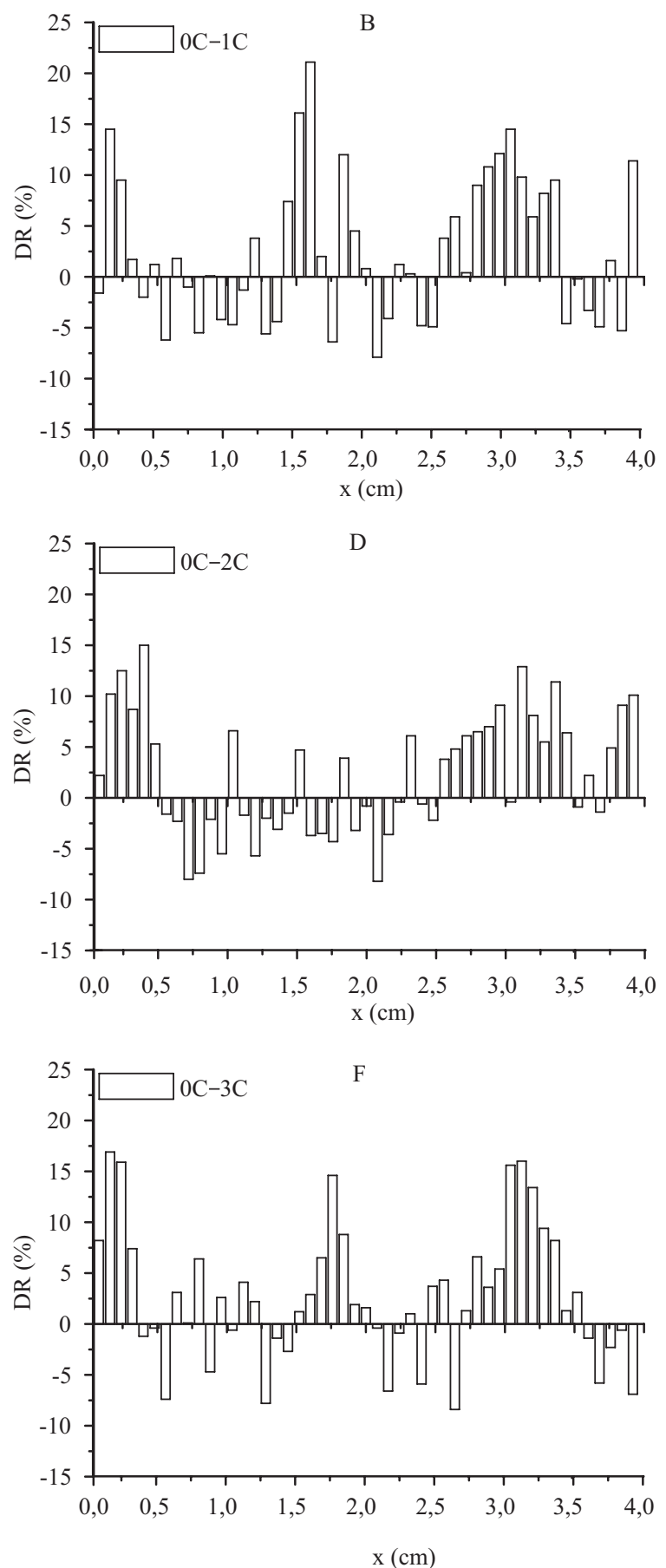

Figura 3. Perfis de densidade média do solo $\left(\rho_{\mathrm{s}}\right)$ para camadas de $0,8 \mathrm{~mm}$ de espessura das amostras de solo. A, amostras submetidas a nenhum (0C) e um (1C) ciclo de umedecimento e secamento (U-S); B, desvio relativo percentual (DR) entre as amostras $0 \mathrm{C}$ e $1 \mathrm{C}$; C, amostras submetidas a $0 \mathrm{C}$ e dois $(2 \mathrm{C})$ ciclos de U-S; D, DR entre as amostras $0 \mathrm{C}$ e $2 \mathrm{C}$; E, amostras submetidas $0 \mathrm{C}$ e três $(3 \mathrm{C}$ ) ciclos de U-S; F, DR entre as amostras $0 \mathrm{C}$ e 3C. O eixo das abscissas indica valores da largura (x) das amostras referentes à linha da matriz de dados de unidades tomográficas selecionada para análise dos perfis de $\rho_{\mathrm{s}}$. 
quando amostras deformadas estão sendo utilizadas na determinação de propriedades hidráulicas do solo (curva de retenção e condutividade hidráulica).

O processo de umedecimento e secamento, em geral, resulta em pequenas alterações no volume total da amostra causadas por estresses em razão de interfaces água-ar, originadas de forças capilares, as quais aumentam com a drenagem do solo. Como consequência, após cada processo de reumedecimento, a estrutura do solo sofrerá novas modificações, e atingirá um novo estado de energia, o qual

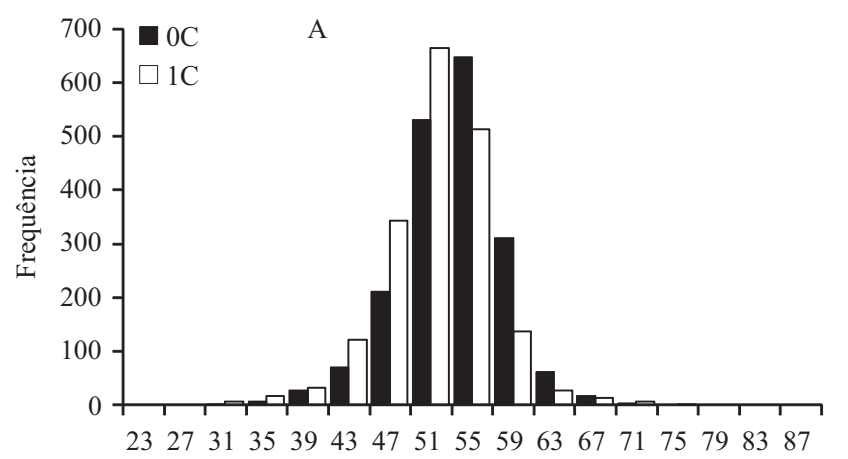

$\phi(\%)$
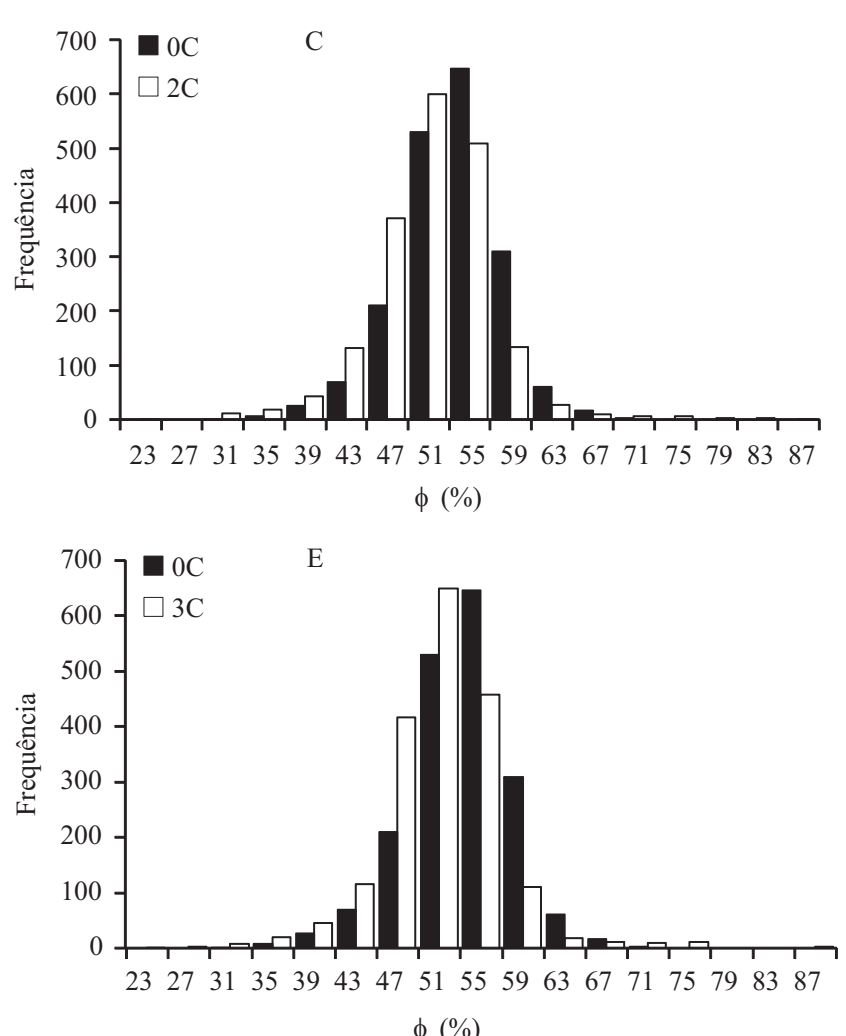

é provavelmente diferente do estado anterior, ocorrido antes do primeiro ciclo de umedecimento e secamento (Baumgartl \& Köck, 2004). Essa nova estrutura do solo não consegue retornar ao estado de energia original, com reflexos e mudanças no sistema poroso do solo (Bresson \& Moran, 2003).

Nas regiões com menor porosidade pela imagem a $\varphi$ já diminui com a aplicação de apenas um ciclo de U-S (Figura 5). Nas imagens de distribuição de $\varphi$ referentes a $1 \mathrm{C}$ até $3 \mathrm{C}$ (Figura $5 \mathrm{~B}, \mathrm{C}$ e D), é visível o aparecimento de
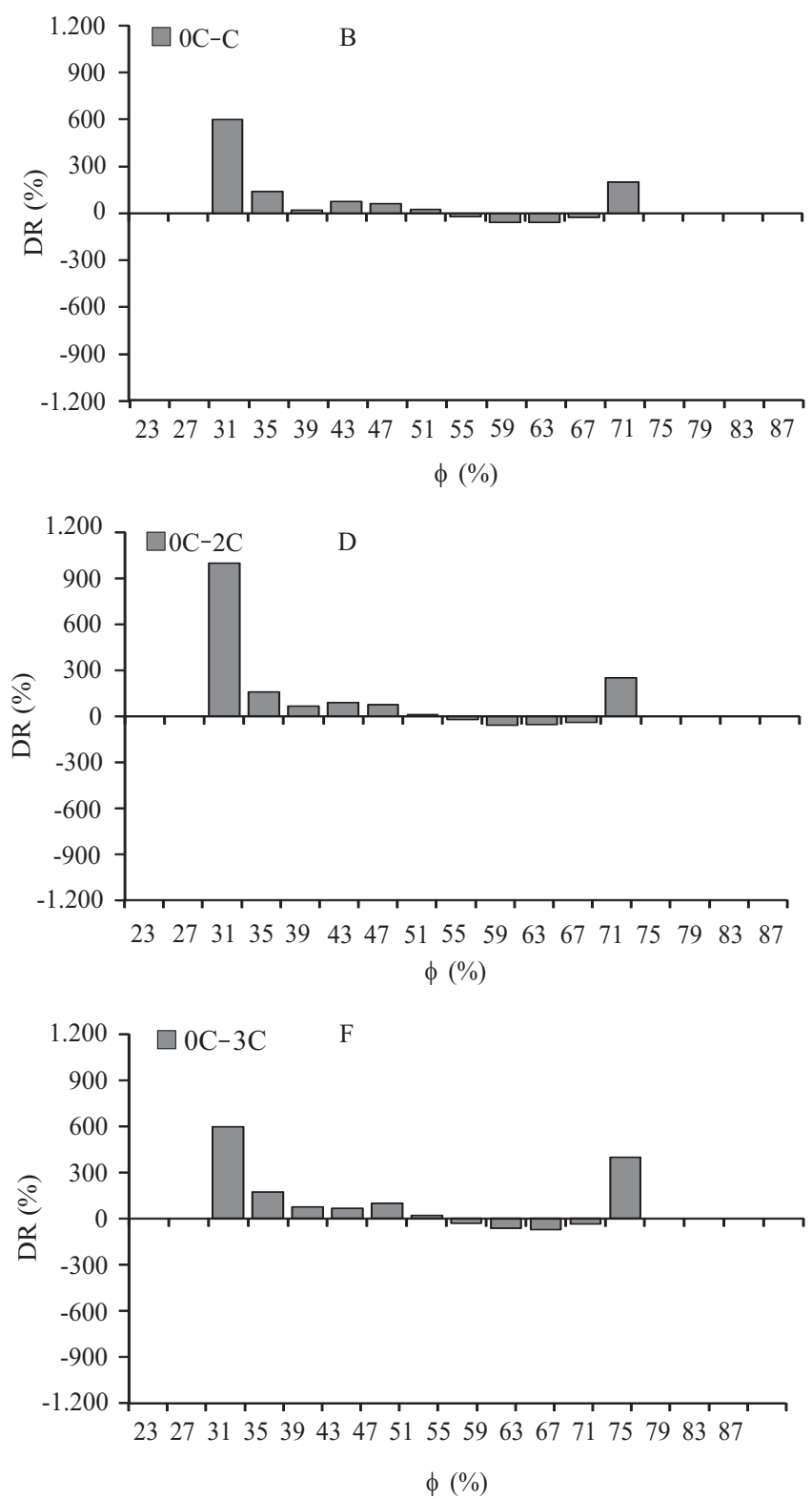

Figura 4. Distribuição de frequência de porosidade pela imagem $(\phi)$ para as amostras de solo avaliadas. A, amostras submetidas a nenhum (0C) e um (1C) ciclo de umedecimento e secamento (U-S); B, desvio relativo percentual (DR) entre as amostras $0 \mathrm{C}$ e $1 \mathrm{C}$; C, amostras submetidas a $0 \mathrm{C}$ e dois $(2 \mathrm{C})$ ciclos de $\mathrm{U}-\mathrm{S}$; D, DR entre as amostras $0 \mathrm{C}$ e 2C; E, amostras submetidas a 0C e três (3C) ciclos de U-S; F, DR entre as amostras 0C e 3C. 

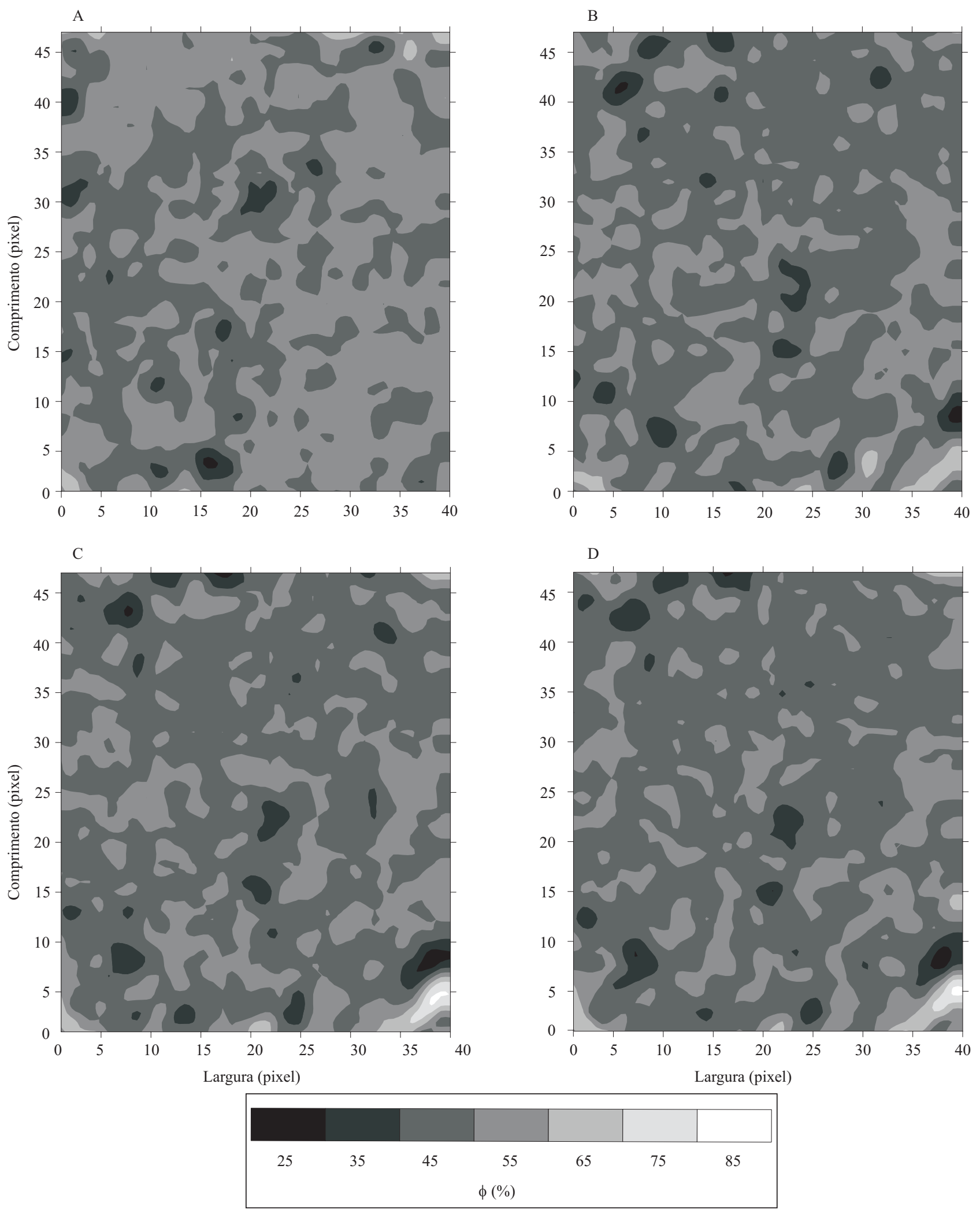

Figura 5. Mapas de variação da porosidade pela imagem $(\phi)$ para as amostras do solo avaliadas. Amostras submetidas a nenhum (A), um (B), dois (C) e três ciclos (D) de umedecimento e secamento. A escala traz os valores de $\phi$ obtidos para uma matriz de dados de 1.880 unidades tomográficas. 
uma região com um grande valor de $\varphi$ no lado inferior direito. Essa região explica o resultado observado nos gráficos de DR (Figuras 4 B, D e F) para a faixa de $\varphi$ de $71-75 \%$. Também é possível observar nos mapas de $\varphi$ que, após aplicação de um ciclo de U-S, quase não existem diferenças perceptíveis entre os mapas para $2 \mathrm{C}$ e $3 \mathrm{C}$ em comparação a $1 \mathrm{C}$. Esse resultado confirma que o maior efeito dos ciclos de U-S em relação às amostras $0 \mathrm{C}$ já ocorre após o primeiro ciclo de umedecimento e secamento. Rajaram \& Erbach (1999) mostraram que somente um único ciclo de U-S pode alterar certos parâmetros físicos do solo, tais como resistência à penetração, tamanho e estabilidade dos agregados.

\section{Conclusões}

1. A técnica de tomografia computadorizada possibilita acompanhar com sucesso, variações na estrutura de amostras deformadas de solos, após serem submetidas a ciclos de umedecimento e secamento.

2. As maiores alterações da estrutura do solo de amostras deformadas ocorrem após o primeiro ciclo de U-S do solo, sendo que os ciclos subsequentes apresentam pequeno efeito sobre as variáveis avaliadas.

\section{Agradecimentos}

Ao Conselho Nacional de Pesquisa e Desenvolvimento, pelo apoio financeiro e concessão de bolsas.

\section{Referências}

BAUMGARTL, T.; KÖCK, B. Modeling volume change and mechanical properties with hydraulic models. Soil Science Society of America Journal, v.68, p.57-65, 2004.

BAVEYE, P.; ROGASIK, H.; WENDROTH, O.; ONASCH, I.; CRAWFORD, J.W. Effect of sampling volume on the measurement of soil physical properties: simulation with X-ray tomography data. Measurement Science and Technology, v.13, p.775-784, 2002.

BRESSON, L.M.; MORAN, C.J. Role of compaction versus aggregate disruption on slumping and shrinking of repacked hardsetting seedbeds. Soil Science, v.168, p.585-594, 2003.

CESAREO, R.; GIANNINI, M. Elemental analysis by means of X-ray attenuation measurements. Nuclear Instruments and Methods, v.169, p.551-555, 1980.

CRESTANA, S.; CRUVINEL, P.E.; VAZ, C.M.P.; CESAREO, R.; MASCARENHAS, S.; REICHARDT, K. Calibração e uso de um tomógrafo computadorizado em ciência do solo. Revista Brasileira de Ciência do Solo, v.16, p.161-167, 1992.

CRESTANA, S.; MASCARENHAS, S.; POZZI-MUCELLI, R.S. Static and dynamic three-dimensional studies of water in soil using computed tomographic scanning. Soil Science, v.140, p.326-332, 1985.

CRUVINEL, P.E.; CRESTANA, S. The use of $X$ and $\gamma$-rays dedicated computerized minitomography scanner in agriculture due to the limitations imposed by medical computerized tomography scanners. IEEE Transactions on Instrumentation and Measurement, p.208-212, 1996.

FERRAZ, E.S.B.; MANSELL, R.S. Determining water content and bulk density of soil by gamma ray attenuation methods. Florida: University of Florida, 1979. 51p.

HAINSWORTH, J.M.; AYLMORE, L.A.G. The use of computerassisted tomography to determine spatial distribution of soil water content. Australian Journal of Soil Research, v.21, p.435-443, 1983.

HERMAN, G.T. Image reconstruction from projections: the fundamentals of computerized tomography. London: Academic Press, 1980. 316p.

HUSSEIN, J.; ADEY, M.A. Changes in microstructure, voids and b-fabric of surface samples of a Vertisol caused by wet/dry cycles. Geoderma, v.85, p.63-82, 1998.

LI, D.; VELDE, B.; ZHANG, T. Observations of pores and aggregates during aggregation in some clay-rich agricultural soils as seen in 2D image analysis. Geoderma, v.118, p.191-207, 2004.

MACEDO, A.; CRESTANA, S.; VAZ, C.M.P. X-ray microtomography to investigate thin layers of soil clod. Soil and Tillage Research, v.49, p.249-253, 1998.

MICROVIS: programa de reconstrução e visualização de imagens tomográficas. São Carlos: Embrapa Instrumentação Agropecuária, 2000. 18p.

PARDINI, G.; VIGNA GUIDI, G.; PINI, R.; REGÜÉS, D.; GALLART, F. Structure and porosity of smectitic mudrocks as affected by experimental wetting-drying cycles and freezingthawing cycles. Catena, v.27, p.149-165, 1996.

PEDROTTI, A.; PAULETTO, E.A.; CRESTANA, S.; CRUVINEL, P.E.; VAZ, C.M.P.; NAIME, J. de M.; SILVA, A.M. da. Tomografia computadorizada aplicada a estudos de um Planossolo. Pesquisa Agropecuária Brasileira, v.38, p.819-826, 2003.

PETROVIC, A.M.; SIEBERT, J.E.; RIEKE, P.E. Soil bulk density analysis in three dimensions by computed tomographic scanning. Soil Science Society of America Journal, v.46, p.445-450, 1982.

PIRES, L.F.; ARTHUR, R.J.C.; BACCHI, O.O.S.; REICHARDT, K. Application of gamma-ray computed tomography to evaluate the radius of influence of soil solution extractors and tensiometers. Nuclear Instruments and Methods in Physics Research Section B: Beam Interactions with Materials and Atoms, v.259, p.969-974, 2007.

RAJARAM, G.; ERBACH, D.C. Effect of wetting and drying on soil physical properties. Journal of Terramechanics, v.36, p.3949, 1999.

REICHARDT, K.; TIMM, L.C. Solo, planta e atmosfera: conceitos, processos e aplicações. Barueri: Manole, 2004. 478p. 
SANTOS, H.G. dos; JACOMINE, P.K.T.; ANJOS, L.H.C. dos; OLIVEIRA, V.A. de; OLIVEIRA, J.B. de; COELHO, M.R.; LUMBRERAS, J.F.; CUNHA, T.J.F. (Ed.). Sistema brasileiro de classificação de solos. 2.ed. Rio de Janeiro: Embrapa Solos, 2006. 306p.

SILVA, A.J.N. da; CABEDA, M.S.V. Influência de diferentes sistemas de uso e manejo na coesão, resistência ao cisalhamento e óxidos de $\mathrm{Fe}$, Si e Al em solo de tabuleiro costeiro de Alagoas. Revista Brasileira de Ciência do Solo, v.29, p.447-457, 2005.

TESSIER, D.; BEAUMONT, A.; PEDRO, G. Influence of clay mineralogy and rewetting rate on clay microstructure. In: DOUGLAS, L.A. (Ed.). Soil micromorphology. Amsterdam: Elsevier Science, 1990. p.115-121.
TIPPKOTTER, R.; EICKHORST, T.; TAUBNER, H.; GREDNER, B.; RADEMAKER, G. Detection of soil water in macropores of undisturbed soil using microfocus X-ray tube computerized tomography $(\mu \mathrm{CT})$. Soil and Tillage Research, v.105, p.12-20, 2009.

VIANA, J.H.M.; FERNANDES FILHO, E.I.; SCHAEFER, C.E.G.R. Efeitos de ciclos de umedecimento e secagem na reorganização da estrutura microgranular de latossolos. Revista Brasileira de Ciência do Solo, v.28, p.11-19, 2004.

VIDAL-TORRADO, P.; LEPSCH, I.F.; CASTRO, S.S.; COOPER, M. Pedogênese em uma seqüência latossolo-podzólico na borda de um platô na depressão periférica paulista. Revista Brasileira de Ciência do Solo, v.23, p.909-921, 1999.

Recebido em 5 de agosto de 2009 e aprovado em 11 de março de 2010 